PROCEEDINGS OF THE

AMERICAN MATHEMATICAL SOCIETY

Volume 51, Number 2, September 1975

\title{
AN INTERMEDIATE VALUE PROPERTY ${ }^{1}$
}

\author{
JOHN L. GAMLEN AND JAMES S. MULDOWNEY
}

ABSTRACT. An intermediate value property is shown to hold for monotone perturbations of maps which have this property. Applications are given to initial value problems and boundary value problems for functional differential equations.

1. Introduction. In [10] basic existence theorems for initial value problems and boundary value problems for ordinary differential equations were expressed in terms of an intermediate value property of maps between partially ordered sets. These results were extended to include functional differential equations where the functional behaviour of the equations satisfied certain monotonicity and continuity requirements. The object of the present paper is to show that the monotonicity requirement alone is sufficient for these results to hold.

2. An intermediate value theorem. Let $\mathcal{X}$ and $\mathcal{S}$ be partially ordered sets. A map $T: X \rightarrow \mathcal{S}$ has the intermediate value property with respect to $s \in \mathcal{S}$ if $\alpha, \beta \in X$ and $\alpha \leq \beta, T \alpha \leq s \leq T \beta$ implies that there exists $z \in \mathcal{S}$ such that $\alpha \leq z \leq \beta$ and $T z=s$.

Theorem. Let $K: \mathcal{X} \times \mathcal{X} \rightarrow \mathcal{S}$ be such that

(i) $K(\cdot, x): X \rightarrow \mathcal{S}$ has the intermediate value property with respect to $s$ for each $x \in \mathcal{X}$.

(ii) $K(x, \cdot): X \rightarrow \mathcal{S}$ is decreasing for each $x \in \mathcal{X}$.

Suppose further that, if $u, v \in X, u \leq v,[u, v]=\{x: u \leq x \leq v\}$ and

$$
X_{s}[u, v]=\{x \in[u, v]: \exists y \in[u, v] \ni K(x, y)=s\}
$$

Received by the editors August 16, 1973 and, in revised form, February 25, 1974.

AMS (MOS) subject classifications (1970). Primary 34A10, 34B15; Secondary 47G05.

Key words and phrases. Intermediate value property, initial value problems, boundary value problems.

1 This paper was written while both authors were on leave from the University of Alberta, Edmonton. Research supported by National Research Council Grant NRC-A7552, Defense Research Board Grant DRB-9540-28 and National Science Foundation Grant NSF-GP 30671. 
(iii) Well-ordered subsets of $X_{s}[u, v]$ have supremums in $X$.

(iv) If $W$ is a well-ordered subset of $X_{s}[u, v]$ such that $\omega=\sup W$ and $K(x, \omega) \leq s$ for each $x \in$ then $K(\omega, \omega) \leq s$.

Then the map $x \rightarrow K(x, x)$ has the intermediate value property with respect to $s$.

Proof. Suppose $\alpha \leq \beta, K(\alpha, \alpha) \leq s \leq K(\beta, \beta)$. Then, from (ii), $K(\alpha, \alpha) \leq$ $s \leq K(\beta, \alpha)$ and (i) implies that there exists $\alpha_{1} \in[\alpha, \beta]$ such that $K\left(\alpha_{1}, \alpha\right)=$ $s$; by (ii) $K\left(\alpha_{1}, \alpha_{1}\right) \leq s$. Thus the set

$$
X_{s}^{*}[\alpha, \beta]=\left\{x \in X_{s}[\alpha, \beta]: K(x, x) \leq s\right\}
$$

is not empty. Let 19 be a well-ordered subset of $X_{s}^{*}[\alpha, \beta]$. If $\omega=\sup 6$ then, from (ii), $K(x, \omega) \leq s$ for each $x \in(1)$ so that condition (iv) implies $K(\omega, \omega) \leq s$. Since $\omega \leq \beta$ and $K(\omega, \omega) \leq s \leq K(\beta, \beta)$, conditions (i) and (ii) imply again that there exists $\omega_{1} \in[\omega, \beta]$ such that $K\left(\omega_{1}, \omega\right)=s$ and $K\left(\omega_{1}, \omega_{1}\right) \leq s$, i.e. $\omega_{1} \in X_{s}^{*}[\alpha, \beta]$. A lemma of Bourbaki [1] states that a partially ordered set has a maximal element if it contains an upper bound for each well-ordered subset. Thus the preceding discussion implies that there exists a maximal element $z \in \mathfrak{X}_{s}^{*}[\alpha, \beta]$; hence $K(z, z) \leq s$. In fact $K(z, z)=$ $s$ since, if $K(z, z)<s$, conditions (i) and (ii) imply that there exists $z_{1}$, $z<z_{1} \leq \beta$, such that $K\left(z_{1}, z\right)=s, K\left(z_{1}, z_{1}\right) \leq s$. Therefore $z_{1} \in \mathfrak{X}_{s}^{*}[\alpha, \beta]$ and this contradiction of maximality proves $K(z, z)=s$.

Remark 1. Note that it has in fact been proved that if (i)-(iv) hold and $\alpha \leq \beta, K(\alpha, \alpha) \leq s \leq K(\beta, \beta)$, then there exists a maximal $z \in[\alpha, \beta]$ such that $K(z, z)=s$.

Remark 2. A similar result to this theorem was proved in [10] subject to additional continuity requirements. A point $z$ was obtained as the limit of a sequence of successive approximations $\left\{z_{n}\right\}$ such that

$$
\alpha=z_{0}, \quad \alpha \leq z_{n} \leq z_{n+1} \leq \beta, \quad K\left(z_{n+1}, z_{n}\right)=s, \quad K\left(z_{n}, z_{n}\right) \leq s .
$$

Remark 3. Condition (ii) may be replaced by the less restrictive condition

(ii) $K(x, y)=s, x \geq y \Rightarrow K(x, x) \leq s ; \quad K(x, x) \geq s, x \geq y \Rightarrow K(x, y) \geq s$.

No change is needed in the proof.

The present theorem, with (ii) replaced by (ii)' ${ }^{\prime}$, includes the following slight extension of a result of Tarski [8].

Corollary 1. Let $T: \mathcal{X} \rightarrow \mathcal{X}$ be increasing. If each well-ordered subset 
of $T \mathcal{X}$ has a supremum in $X$, then $\alpha \leq T \alpha \leq T \beta \leq \beta$ implies there exists a maximal $z \in[\alpha, \beta]$ such that $T z=z$.

Proof. Let $\mathcal{S}=\{-1,0,1, i\}$ be partially ordered by $-1<0<1$. Define $K: \mathcal{X} \times \mathfrak{X} \rightarrow \mathcal{S}$ by

$$
\begin{aligned}
K(x, y) & =-1, \quad \text { if } x<T y \\
& =0, \quad \text { if } x=T y \\
& =1, \quad \text { if } x>T y, \\
& =i, \quad \text { if } x \text { and } T y \text { are incomparable. }
\end{aligned}
$$

$K$ satisfies conditions (i), (ii)' with $s=0 . X_{0}[u, v] \subset T X$ for each $[u, v] \subset X$ and condition (iii) is satisfied by hypothesis; if $\mathcal{W}$ is a well-ordered subset of $T \mathcal{X}$ with $\omega=\sup \mathcal{W}$ and $x \leq T \omega$ for each $x \in \mathcal{W}$, then $\omega \leq T \omega$ so that condition (iv) holds.

A generalization of Tarski's theorem in a direction different from the present theorem is due to Wong [11] and also includes Corollary 1 as a special case when it is assumed that well-ordered subsets of $\mathcal{X}$ have supremums. The proof in [11] may be easily modified to include Corollary 1 completely. In the case that $\mathcal{X}$ is the space of real Lebesgue measurable functions of a real variable, Corollary 1 leads to generalizations of integral inequalities of the Gronwall type (cf. Hanson and Waltman [4] and Walter [9, Chapter I]). A generalization of Gronwall's inequality in which an integral operator $T$ does not satisfy the monotonicity condition of Corollary 1 may be found in [10, Theorem 4.1].

The following example shows that the semicontinuity condition (iv) on $K(\cdot, y)$ cannot be omitted.

Example 1. Let $X=[0,1]$ and $\mathcal{S}=\{0, s, 1\}$ where $\mathcal{S}$ is ordered by $0<$ $s<1$. Partition $[0,1]$ into two disjoint subsets $P$ and $Q$ each dense in $[0,1]$, and let

$$
\begin{aligned}
& K(x, y)=1, \quad \text { if } x \in P, y \leq x \text {, } \\
& =s, \text { if } x \in P, y>x \text {, } \\
& s, \quad \text { if } x \in Q, y<x \text {, } \\
& =0, \quad \text { if } x \in Q, y \geq x \text {. }
\end{aligned}
$$

Condition (i) holds since $s \in K(I, y)$ for each interval $I \subset[0,1]$ and each $y \in[0,1]$. Evidently $K(x, \cdot)$ is decreasing for each $x$ so condition (ii) is satisfied and condition (iii) holds from the completeness of $[0,1]$. Never- 
theless the map $x \rightarrow K(x, x)$ does not have the intermediate value property with respect to $s$, since $K(x, x)=0$ or 1 for each $x$. Condition (iv) is not satisfied at any point $\omega \in P$; each such point is the supremum of a sequence $\left\{x_{n}\right\}$ for which $K\left(x_{n}, \omega\right)=s$ while $K(\omega, \omega)=1>s$.

An example similar to Example 1 in which $\mathcal{X}=\mathcal{S}=[0,1]$ for which conditions (i), (ii), (iii) are satisfied while the map $x \rightarrow K(x, x)$ fails to have the intermediate value property with respect to each $s \in(0,1)$ may be found as follows. Let $f:[0,1] \rightarrow[0,1]$ be such that $f(I)=[0,1]$ for each subinterval $I$ of $[0,1]$ (cf. $\left[3\right.$, p. 31]). Thus $f^{-1}(s)$ is dense in $[0,1]$ for each $s \in[0,1]$. Let $f^{-1}(s)=P_{s} \cup Q_{s}$, where $P_{s}$ and $Q_{s}$ are disjoint sets each dense in $[0,1]$, and define $K:[0,1]^{2} \rightarrow[0,1]$ as in Example 1 with $\{P, Q\}$ replaced by $\left\{P_{s}, Q_{s}\right\}$ for each $s \in[0,1]$.

3. Applications. A function $f: I \times R \rightarrow R$, where $I$ is an interval in $R$, is said to satisfy Carathéodory's conditions if $f(t, \cdot): R \rightarrow R$ is continuous for each $t \in I, f(\cdot, x): I \rightarrow R$ is Lebesgue measurable for each $x \in R$ and, for each compact subset $S \subset R$, there exists $m \in$ loc $L^{1}(I)$ such that $|f(t, x)| \leq m(t)$, for each $x \in S$. Suppose $f$ satisfies Carathéodory's conditions on $[0, b) \times R$, where $0<b \leq \infty$, and $\alpha, \beta \in \operatorname{loc} A C[0, b)$ are such that

$$
\begin{array}{ll}
\alpha^{\prime}(t) \leq f(t, \alpha(t)), & \beta^{\prime}(t) \geq f(t, \beta(t)), \quad \text { a.e. } t \in[0, b), \\
\alpha(0) \leq z_{0} \leq \beta(0), & \alpha(t) \leq \beta(t), \quad t \in[0, b)
\end{array}
$$

then there exists a solution of $z^{\prime}(t)=f(t, z(t)), z(0)=z_{0}$, such that $\alpha(t) \leq$ $z(t) \leq \beta(t), t \in[0, h)$ (cf. [2, p. 30]). This means that the operator $T$ : loc $A C[0, b) \rightarrow$ loc $L^{1}[0, h) \times R$ has the intermediate value property with respect to $\left(0, z_{0}\right)$, where

$$
(7 x)(t)=\left(x^{\prime}(t)-f(t, x(t)), x(0)\right) .
$$

The following result is an immediate consequence of this observation together with Theorem 1 .

Corollary 2. Let $f:[0, h) \times R \times \operatorname{loc} A C[0, b) \rightarrow R$ be such that

(i) $f(t, x, y(\cdot))$ satisfies Carathéodory's conditions in $(t, x)$ for each $y \in \operatorname{loc} A C[0, h)$,

(ii) $f(t, x, y(\cdot))$ is increasing in $y(\cdot)$ for each $(t, x) \in[0, b) \times R$. Suppose $\alpha, \beta \in \operatorname{loc} A C[0, b)$ satisfy

$$
\begin{aligned}
& \alpha^{\prime}(t) \leq f(t, \alpha(t), \alpha(\cdot)), \quad \beta^{\prime}(t) \geq f(t, \beta(t), \beta(\cdot)) \quad \text { a.e. } t \in[0, b), \\
& \alpha(0) \leq z_{0} \leq \beta(0), \quad \alpha(t) \leq \beta(t), \quad t \in[0, b) .
\end{aligned}
$$


Then there exists a solution of the functional differential equation

$$
z^{\prime}(t)=f(t, z(t), z(\cdot)), \quad z(0)=z_{0},
$$

such that $a(t) \leq z(t) \leq \beta(t), t \in[0, h)$. Furthermore there exists a maximal element in the set of solutions $z, \alpha \leq z \leq \beta$.

In this case let

$$
\begin{aligned}
& X=\operatorname{loc} A C[0, b), \quad \mathcal{S}=\operatorname{loc} L^{1}[0, b) \times R, \\
& K(x, y)(t)=\left(x^{\prime}(t)-f(t, x(t), y(\cdot)), x(0)\right) .
\end{aligned}
$$

Conditions (i) and (ii) of the Theorem are satisfied by $K$. For each $u, v \in X$, $u \leq v$, the (possibly empty) set $X_{\left(0, z_{0}\right)}[u, v]$ of solutions $x \in X_{\text {to initial }}$ value problems $x^{\prime}(t)=f(t, x(t), y(\cdot)), x(0)=z_{0}$, where $u \leq y \leq v$, which satisfy $u \leq x \leq v$ is uniformly bounded and equi-absolutely continuous locally on $[0, h)$. If $W$ is a well-ordered subset of $X_{\left(0, z_{0}\right)}[u, v]$ there is a sequence $\left\{x_{n}\right\} \subset \mathcal{W}$ such that $x_{n} \uparrow \omega=$ sup $W$; the convergence is uniform on compact subsets of $[0, b)$ and hence $\omega \in \mathcal{X}$, i.e. (iii) holds. If each $x \in \mathcal{1 0}$ satisfies $x^{\prime}(t) \leq f(t, x(t), \omega(\cdot)), x(0)=z_{0}$, or equivalently

$$
x\left(t_{2}\right)-x\left(t_{1}\right) \leq \int_{t_{1}}^{t_{2}} f(s, x(s), \omega(\cdot)) d s, \quad x(0)=z_{0},
$$

if $0 \leq t_{1}<t_{2}<h$, then the Lebesgue dominated convergence theorem applied to $\left\{x_{n}\right\}$ implies

$$
\omega\left(t_{2}\right)-\omega\left(t_{1}\right) \leq \int_{t_{1}}^{t_{2}} f(s, \omega(s), \omega(\cdot)) d s, \quad \omega(0)=z_{0},
$$

for each $\left[t_{1}, t_{2}\right] \subset[0, b)$. Since $\omega \in \mathcal{X}$ this statement is equivalent to $\omega^{\prime}(t) \leq f(t, \omega(t), \omega(\cdot)), \omega(0)=z_{0}$, so condition (iv) is satisfied.

In a similar manner the following result of Jackson and Schrader [6] yields Corollary 3. If $f:[0,1] \times R \rightarrow R$ satisfies Carathéodory's conditions and $\alpha, \beta \in A C^{(1)}[0,1]$ satisfy

$$
\begin{aligned}
& \alpha^{\prime \prime}(t) \geq f(t, \alpha(t)), \quad \beta^{\prime \prime}(t) \leq f(t, \beta(t)) \quad \text { a.e. } t \in[0,1] \text {, } \\
& \alpha(0) \leq z_{0} \leq \beta(0), \quad \alpha(1) \leq z_{1} \leq \beta(1), \quad \alpha(t) \leq \beta(t), \quad t \in[0,1],
\end{aligned}
$$

then there exists a solution $z(t)$ of the boundary value problem $z^{\prime \prime}(t)=$ $f(t, z(t)), z(0)=z_{0}, z(1)=z_{1}$, such that $\alpha(t) \leq z(t) \leq \beta(t), t \in[0,1]$.

Corollary 3. Let $f:[0,1] \times R \times A C^{(1)}[0,1] \rightarrow R$ be such that 
(i) $f(t, x, y(\cdot))$ satisfies the Carathéodory conditions in $(t, x)$ for each $y \in A C^{(1)}[0,1]$,

(ii) $f(t, x, y(\cdot))$ is decreasing in $y$ for each $(t, x) \in[0,1] \times R$. If $\alpha, \beta \in A C^{(1)}[0, h]$ are such that

$$
\begin{aligned}
& \alpha^{\prime \prime}(t) \geq f(t, \alpha(t), \alpha(\cdot)), \quad \beta^{\prime \prime}(t) \leq f(t, \beta(t), \beta(\cdot)) \quad \text { a.e. } t \in[0,1], \\
& \alpha(0) \leq z_{0} \leq \beta(0), \quad \alpha(1) \leq z_{1} \leq \beta(1), \quad \alpha(t) \leq \beta(t), \quad t \in[0,1],
\end{aligned}
$$

then there exists a maximal solution of the functional differential boundary value problem

$$
z^{\prime \prime}(t)=f(t, z(t), z(\cdot)), \quad z(0)=z_{0}, \quad z(1)=z_{1},
$$

such that $a(t) \leq z(t) \leq \beta(t), t \in[0,1]$.

This follows from consideration of

$$
K(x, y)(t)=\left(f(t, x(t), y(\cdot))-x^{\prime \prime}(t), x(0), x(1)\right) .
$$

Corollary 3 holds also if $f(t, x, y(\cdot))$ is replaced by $f\left(t, x, x^{\prime}, y(\cdot)\right)$ provided $f$ satisfies a Nagumo condition in $x^{\prime}$ for each $y$.

The technique described here and in [10] seems to be particularly suited to the study of certain singular boundary value problems arising in boundary layer theory. For illustrative purposes one of the simplest problems of this type is discussed in Example 2 (cf. [5, pp. 521-523]).

Example 2. The boundary value problem

$$
\begin{aligned}
& u^{\prime \prime \prime}+u u^{\prime \prime}+\lambda\left(1-u^{\prime 2}\right)=0, \\
& u(0)=a, \quad u^{\prime}(0)=b, \quad u^{\prime}(\infty)=1,
\end{aligned}
$$

where $\lambda \geq 0,0<b<1$, has a solution.

This problem is equivalent to the second order functional differential boundary value problem

$$
z^{\prime \prime}(t)=f\left(t, z(t), z^{\prime}(t), z(\cdot)\right), \quad z(0)=b, \quad z(\infty)=1,
$$

where $f\left(t, x, x^{\prime}, y(\cdot)\right)=-\left(a+\int_{0}^{t} y\right) x^{\prime}-\lambda\left(1-x^{2}\right)$. By a result of Jackson [7, Theorem 7.4] (a singular version of the result in [6] quoted for Corollary 3),

$$
K(x, y)(t)=\left(f\left(t, x(t), x^{\prime}(t), y(\cdot)\right)-x^{\prime \prime}(t), x(0)\right)
$$

has the intermediate value property in $x$ with respect to $(0, b)$ for each $y$ in the space $\operatorname{loc} L^{1}[0, \infty) ; K(x, y)$ is decreasing in $y$ if $x^{\prime} \geq 0$. Consider

$$
\beta(t)=1, \quad \alpha(t)=(1-b) I^{-1} \int_{0}^{t} \exp \left(-\int_{0}^{s} r\right) d s+b, \quad t \in[0, \infty),
$$


where $r(t)=a+b t$ and $I=\int_{0}^{\infty} \exp \left(-\int_{0}^{s} r\right) d s$. These functions satisfy $b \leq$ $a(t) \leq \beta(t)=1$,

$$
\begin{aligned}
& \alpha^{\prime \prime}(t) \geq f\left(t, \alpha(t), \alpha^{\prime}(t), \alpha(\cdot)\right), \quad \alpha^{\prime}(t) \geq 0, \quad \alpha(0)=b, \quad \alpha(\infty)=1, \\
& \beta^{\prime \prime}(t)=f\left(t, \beta(t), \beta^{\prime}(t), \beta(\cdot)\right), \quad \beta^{\prime}(t)=0, \quad \beta(0)=\beta(\infty)=1
\end{aligned}
$$

because $f(t, 1,0,1(\cdot))=0$ and

$$
\alpha^{\prime \prime}=-r(t) \alpha^{\prime}=-(a+b t) \alpha^{\prime} \geq-\left(a+\int_{0}^{t} \alpha\right) \alpha^{\prime}-\lambda\left(1-\alpha^{2}\right)=f\left(t, \alpha, \alpha^{\prime}, \alpha(\cdot)\right)
$$

since $0<b \leq a \leq 1, \alpha^{\prime} \geq 0, \lambda \geq 0$. Furthermore solutions of $z^{\prime \prime}=$ $f\left(t, z, z^{\prime}, y(\cdot)\right), z(0)=b, z(\infty)=1$ with $\alpha(t) \leq z(t) \leq 1$ satisfy $z^{\prime}(t) \geq 0$ because $z^{\prime}\left(t_{0}\right)<0$ implies $z^{\prime}(t)<0, t \geq t_{0}$ (and hence $z(\infty)<1$ ), from

$$
z^{\prime \prime}+\left(a+\int_{0}^{t} y\right) z^{\prime}=-\lambda\left(1-z^{2}\right) \leq 0
$$

By choosing $X=\left\{x \in \operatorname{loc} A C^{1}[0, \infty): \alpha \leq x \leq 1, x^{\prime} \geq 0\right\}$ it follows that $K$ satisfies conditions (i), (ii) of the Theorem, and $\mathscr{X}_{(0, b)}[u, v]$ satisfies (iii), (iv) for each $u, v \in X, u \leq v$. Thus (P) has a solution in $X$.

\section{REFERENCES}

1. N. Bourbaki, Sur le théorème de Zorn, Arch. Math. 2 (1949/50), 434-437 (1951). MR 13, 923.

2. W. A. Coppel, Stability and asymptotic behavior of differential equations, Heath, Boston, Mass., 1965. MR 32 \#7875.

3. B. R. Gelbaum and M. H. Olmsted, Counterexamples in analysis, The Mathesis Series, Holden-Day, San Francisco, Calif., 1964. MR 30 \#204.

4. D. L. Hanson and P. Waltman, A note on a functional equation, J. Math. Anal. Appl. 10 (1965), 330-333. MR 30 \#4079.

5. Philip Hartman, Ordinary differential equations, Wiley, New York, 1964. MR $30 \# 1270$.

6. Lloyd K. Jackson and Keith W. Schrader, Comparison theorems for nonlinear differential equations, J. Differential Equations 3 (1967), 248-255. MR 34 \#6206.

7. Lloyd K. Jackson, Subfunctions and second-order ordinary differential inequalities, Advances in Math. 2 (1968), 307-363. MR 37 \#5462.

8. Alfred Tarski, A lattice-theoretical fixpoint theorem and its applications, Pacific J. Math. 5 (1955), 285-309. MR 17, 574.

9. Walter Wolfgang, Differential and integral inequalities, Ergebnisse der Mathematik und ihrer Grenzgebiete, Band 55, Springer-Verlag, New York and Berlin, 1970. MR $42 \# 6391$.

10. D. Willett and J. S. Muldowney, An intermediate value property for operators with applications to integral and differential equations, Canad. J. Math. 26 (1974), 27-41. 
11. James S. W. Wong, Common fixed points of commuting monotone mappings, Canad. J. Math. 19 (1967), 617-620. MR 35 \#1513.

DEPARTMENT OF MATHEMATICS, YALE UNIVERSITY, NEW HAVEN, CONNECTICUT 06520

DE PAR TMENT OF MATHEMATICS, UNIVERSITY OF UTAH, SALT LAKE CITY, UTAH 84112

Current address (both authors): Department of Mathematics, University of Alberta, Edmonton, Alberta, Canada T6G 2G1 